\section{Dimethyl fumarate as a safe and effective therapy for recalcitrant psoriasis in comorbid patients}

Martina Burlando, Mattia Fabio Molle,
Emanuele Cozzani, Aurora Parodi

Section of Dermatology, DISSAL, San Martino-IST Polyclinic Hospital, University of Genoa, Italy

\begin{abstract}
Psoriasis is a chronic condition for which multiple therapies are currently available. In particular, in cases of moderate-severe psoriasis, traditional systemic drugs or the new biological drugs can be administered. However, the treatment of patients who require systemic therapy and have multiple comorbidities can be particularly complex. Some treatment options may be in fact contraindicated or may lose effectiveness over time, reducing the options available to the dermatologists. In such circumstances, dimethyl fumarate may represent a safe and effective choice, also in patients who have already attempted biological therapies. In this regard, we report the case of a patient with moderate-severe psoriasis treated over time with various therapies (including topicals, phototherapy, traditional and biological drugs) that were discontinued due to ineffectiveness or incompatibility caused by the occurrence of concomitant diseases, who finally achieved clinical remission with dimethyl fumarate.
\end{abstract}

\section{Introduction}

Psoriasis is a chronic, immune-mediated, inflammatory disease characterized by the presence of indurated erythematous plaques covered by silvery desquamative scales that usually involve knees, elbows, trunk and scalp. ${ }^{1}$ Nowadays there are multiple therapeutic options available for the treatment of psoriasis, including topicals, phototherapy, traditional systemic and novel biologic agents. ${ }^{2}$ Systemic therapies used in severe forms of disease are based on principles with immunosuppressive or immunomodulating activity, so it can be complex in patients with multiple underlying comorbidities to find the best therapy. We present the case of a psoriatic patient with multiple pathologies and numerous previous treatments in which it was finally possible to obtain an excellent clinical con- trol of the disease thanks to the use of dimethyl fumarate (DMF).

\section{Case Report}

A 57-year-old Caucasian man has been followed at our Dermatology Clinic since 2005 for plaque psoriasis with prevalent localization to the scalp, elbows, knees, inguinal and intergluteal folds. In his medical history, the patient presented chronic hepatitis $\mathrm{C}$ virus (HCV) infection.

Until 2012, the patient experienced good clinical control of the disease through the application of topical products (steroids and vitamin D analogues) together with balneophototherapy and periodic cycles of nbUVB phototherapy.

In February 2013, the patient presented a marked clinical worsening characterized by numerous infiltrated erythematousdesquamative plaques on the trunk and limbs, with a Psoriasis Area Severity Index (PASI) score of 16. After hepatological consultation, a treatment with cyclosporine at the initial dose of $2.5 \mathrm{mg} / \mathrm{kg} /$ day was therefore started with immediate clinical response. However, after 4 months, therapy was discontinued because of an increase in serum creatinine and the patient returned to topical treatments. After several months of good clinical response, the patient had a new relapse in September 2014, with involvement of the scalp, trunk, limbs, and nail onycholysis of the hands and feet. The patient denied joint pain. At the same time, the patient tested positive to the QuantiFERON-TB Gold test and showed certain pulmonary nodules on chest X-ray. A pneumological diagnostic work-up was carried out, including bronchoalveolar lavage and microscopic and cultural examinations, which excluded the presence of Mycobacterium tuberculosis strains or malignant tumor cells. Therefore, after hepatologic consultation and the initiation of prophylactic therapy with isoniazid, the patient in February 2015 began a therapy with etanercept. At follow-up blood tests in May 2015, the patient unfortunately showed a significant increase in liver function indices so etanercept and isoniazid were discontinued, and the patient returned again to topical treatments. Meanwhile, in 2016, the patient began direct acting antiviral treatment for $\mathrm{HCV}$ with excellent response and normalization of liver function. Given the further worsening of psoriasis, etanercept in combination with topical treatments was started again in December 2016. On that occasion, the patient did not resume antituberculous prophylactic therapy due to
Correspondence: Martina Burlando, Section of Dermatology, DISSAL, San Martino-IST Polyclinic Hospital, University of Genoa, 16132 Genoa, Italy.

Tel.: +393491029766

E-mail: martinaburlando@hotmail.com

Key words: Psoriasis, Dimethyl fumarate, Comorbidities, Biologics, Malignancy.

Contributions: Study conception and design: MB, MFM, EC, AP. Acquisition of data: MB, MFM. Drafting of the manuscript: MFM. Critical revision: $\mathrm{MB}, \mathrm{EC}, \mathrm{AP}$.

Conflict of interest: The authors declare no potential conflict of interest.

Funding: None.

Ethics: All the procedures adopted in the present study were in respect to the ethical standards in the World Medical Association Declaration of Helsinki. The subject gave his written informed consent to publish the present case (including publication of images).

Please cite this article as: Burlando M, Molle MF, Cozzani E, Parodi A. Dimethyl fumarate as a safe and effective therapy for recalcitrant psoriasis in comorbid patients. Dermatol Rep 2022;14:9091.

Received for publication: 30 January 2021. Accepted for publication: 26 April 2021.

This work is licensed under a Creative Commons Attribution-NonCommercial 4.0 International License (CC BY-NC 4.0).

${ }^{\circ}$ Copyright: the Author(s), 2022

Licensee PAGEPress, Italy

Dermatology Reports 2022; 14:9091

doi:10.4081/dr.2022.9091

previous intolerance, and we opted for close follow-up visits. After an initial positive response, the patient experienced progressive secondary inefficacy so in June 2017 he was switched to adalimumab with immediate skin clearance. Unfortunately, the patient had at the same time a new progressive worsening of liver function and was listed for liver transplantation. In March 2018 , following the finding of a liver nodule suggestive of malignancy, adalimumab therapy was discontinued. In April 2018, the patient underwent liver resection with a diagnosis of hepatocarcinoma. The psoriasis relapsed again; this time poorly responsive to topical treatments. Therefore, in September 2018, a therapy with apremilast was initiated, with modest results. Given the persistence of diffuse lesions after six months of therapy (PASI 10), a new cycle of 
nb-UVB phototherapy was associated. In May 2019, the psoriasis had further worsened with a PASI of 13 . We therefore decided to start a therapy with DMF, recently available in Italy, at increasing dosage as per drug data sheet.

To date, the patient is taking DMF $600 \mathrm{mg} /$ day, with good tolerability and complete clinical resolution of psoriasis.

\section{Discussion}

Chronic plaque psoriasis is the most common form of psoriasis, affecting about $90 \%$ of patients. Nowadays, as a consequence of a better understanding of the pathogenetic mechanisms of the disease, many therapeutic options have become available ${ }^{2}$. Nevertheless, finding the right therapy, especially in complex patients, can still be a challenging task. In moderate to severe psoriasis, the use of systemic therapy is recommended.

In some European countries, fumaric acid esters (FAEs) such as Fumaderm ${ }^{\circledR}$, a combination of DMF and monoethyl fumarate salts, have been used for years as a systemic therapy for psoriasis. ${ }^{3}$ In this combination, DMF is believed to be the primary active ingredient and responsible for efficacy in the treatment of psoriasis. Therefore in 2017, the European Medicines Agency (EMA) approved Skilarence ${ }^{\circledR}$, a new oral formulation of DMF for the treatment of adults with moderate-to-severe chronic plaque psoriasis in need of systemic medical therapy. ${ }^{2}$ The safety profile and efficacy of DMF were investigated by the BRIDGE trial, ${ }^{4}$ whereas other previous studies had already investigated the efficacy of FAEs in the treatment of psoriasis. ${ }^{3}$

In our patient's case, the existence of important comorbidities limiting the therapeutic range and the failure of multiple ther- apies have made the management of his psoriasis particularly complex.

Moreover, the treatment of psoriasis in patients with cancer history is especially difficult: malignancy represents at least a relative contraindication to the use of immunosuppressive drugs such as methotrexate or cyclosporine, while the use of biologics is generally considered with caution in patients that have been cancerfree for at least 5 years. ${ }^{5}$ Data in the literature appear to indicate an increased risk of certain malignancies in patients with psoriasis, especially lymphohematopoietic, head and neck, and gastrointestinal tract cancers. There is also an increased risk of nonmelanoma skin cancers (NMSC), possibly as a result of prior therapies such as p-UVA phototherapy or cyclosporine. With regard to biologic drugs, some studies suggest a possible increased risk of NMSC in subjects treated with anti-TNF alpha, while there are no reports about the other molecules. ${ }^{6}$ However, there is a lack of long-term safety data regarding newer biologic agents, which leads to a very cautious use of them in patients with a history of cancer. This also tends to cause patients to be managed with topical therapies that are less effective in moderate-severe psoriasis, with a detriment of quality of life.

In this multifaceted scenario, DMF could represent a valid therapeutic choice in terms of safety. In our experience, in fact, DMF is an effective treatment option, generally well tolerated, with few side effects that recede by adjusting the dose or by discontinuation of the drug.

\section{Conclusions}

This case is of interest because in patients with many comorbidities, there is a tendency to use only topical therapies or phototherapy even in cases of moderatesevere psoriasis, with deterioration in the patient's quality of life. Thus, DMF is a valid and safe therapeutic option in patients requiring systemic therapy either as a first line or in case of previous unsuccessful treatments.

\section{References}

1. Burlando M, Molle MF, Antonelli CT et al. Erythema multiforme after initiation of anti-interleukin-12/23 (ustekinumab) treatment for plaque psoriasis. JAAD Case Rep 2020;6:386-7.

2. Mrowietz U, Barker J, Boehncke WH et al. Clinical use of dimethyl fumarate in moderate-to-severe plaque-type psoriasis: a European expert consensus. J Eur Acad Dermatol Venereol 2018;32:3-14.

3. Blair HA. Dimethyl Fumarate: A Review in Moderate to Severe Plaque Psoriasis. Drugs 2018;78:123-30.

4. Mrowietz U, Szepietowski JC, Loewe $\mathrm{R}$, et al. Efficacy and safety of LAS41008 (dimethyl fumarate) in adults with moderate-to-severe chronic plaque psoriasis: a randomized, doubleblind, Fumaderm ${ }^{\circledR}$ - and placebo-controlled trial (BRIDGE). Br J Dermatol 2017;176:615-23. Erratum in: $\mathrm{Br} \mathrm{J}$ Dermatol 2018;178:308.

5. Takeshita J, Grewal S, Langan SM, et al. Psoriasis and comorbid diseases: Implications for management. J Am Acad Dermatol 2017;76:393-403.

6. Elmets CA, Leonardi CL, Davis DMR, et al. Joint AAD-NPF guidelines of care for the management and treatment of psoriasis with awareness and attention to comorbidities. J Am Acad Dermatol 2019;80:1073-113. 